\title{
Single-Cycle Nonlinear Optics
}

E. Goulielmakis, M. Schultze, M. Hofstetter,V. S. Yakovlev, J. Gagnon, M. Uiberacker, A. L. Aquila, E. M. Gullikson, D. T. Attwood, R. Kienberger, F. Krausz, U. Kleineberg

Max-Planck-Institut für Quantenoptik, Hans-Kopfermann-Strasse 1, D-85748 Garching, Germany

Department fur Physik, Ludwig-Maximilians-Univeresitat, Am Coulombwall 1, D85748 Garching.

Center for X-ray Optics, Lawrence Berkeley National Laboratory, Berkeley, CA 94720, USA

This work was supported by the Director, Office of Science, Office of Basic Energy Sciences, of the U.S. Department of Energy under Contract No. DEAC02-05CH11231. 


\section{Single-Cycle Nonlinear Optics}

E. Goulielmakis ${ }^{1 *}$, M. Schultze ${ }^{1}$, M. Hofstetter ${ }^{2}$, V. S. Yakovlev ${ }^{2}$, J. Gagnon ${ }^{1}$, M. Uiberacker $^{2}$, A. L. Aquila ${ }^{3}$, E. M. Gullikson ${ }^{3}$, D. T. Attwood ${ }^{3}$, R. Kienberger ${ }^{1}$, F. Krausz $^{1,2 *}$, U. Kleineberg ${ }^{2 *}$

${ }^{1}$ Max-Planck-Institut für Quantenoptik, Hans-Kopfermann-Str. 1, D-85748 Garching, Germany.

${ }^{2}$ Department für Physik, Ludwig-Maximilians-Universität, Am Coulombwall 1, D-85748 Garching.

${ }^{3}$ Center for X-Ray Optics, Lawrence Berkeley National Laboratory, Berkeley, CA 94720, USA.

Abstract: Nonlinear optics plays a central role in the advancement of optical science and laser-based technologies. We report on the confinement of the nonlinear interaction of light with matter to a single wave cycle and demonstrate its utility for time-resolved and strong-field science. The electric field of 3.3femtosecond, $0.72-\mu \mathrm{m}$ laser pulses with a controlled and measured waveform ionizes atoms near the crests of the central wave cycle, with ionization being virtually "switched off" outside this interval. Isolated sub-100-attosecond pulses of extreme ultraviolet light (photon energy $\sim 80 \mathrm{eV}$ ), containing nearly a nanojoule of energy, emerge from the interaction with a conversion efficiency of $\sim 10^{-6}$. These tools enable one to study the precision control of electron motion with light fields and electron-electron interactions with a resolution approaching the atomic unit of time ( 24 as).

Nonlinear electron-light interactions driven by strong light fields of controlled waveform (1) have allowed for the control of electronic motion at light frequencies and the real-time observation of electron dynamics inside and between atoms with $\sim 100$-attosecond resolution (2-7). However, time-domain access to a number of fundamental processes such as the intra-atomic energy transfer between electrons (8), the response of an atomic electron system to external influence (9), and its rearrangement following the sudden loss of one or more

\footnotetext{
"To whom correspondence should be addressed: elgo@mpq.mpg.de, krausz@lmu.de, ulf.kleineberg@physik.unimuenchen.de.
} 
electrons (10), or by the charge transfer in biologically-relevant molecules (11) and related changes in chemical reactivity (12), or due to non-adiabatic tunneling $(13,14)$, would require (or benefit from) an improved temporal resolution.

By using waveform-controlled sub-1.5-cycle near-infrared (NIR) light, we demonstrate the generation of robust, energetic, isolated sub-100-as pulses of extreme ultraviolet (XUV) radiation and their precise temporal characterization. Photoionization confined to a single wave cycle results in observables (such as high-harmonic photons and electrons emitted by above-threshold ionization) that can now be related to several distinguishable sub-cycle ionization events and subsequent electron trajectories with a known timing with respect to the driving field, whose strength and temporal evolution is accurately known (3). These circumstances provide ideal conditions for testing models of strong-field control of electron motion and electron-electron interactions.

The generation of attosecond pulses benefits from the abrupt onset of ionization within a single half-cycle, which minimizes the density of free electrons, and hence the distortion of the driving wave and its dephasing with the generated harmonic wave. As a result, the coherent build-up of the harmonic emission over an extended propagation is maximized. In addition, the order-of-magnitude variation of the ionization probability between adjacent half-cycles creates unique conditions for single sub-100 as pulse emission without the need for sophisticated gating techniques $(5,15,16)$.

On the measurement side, improved resolution results from three provisions: shorter XUV pulse duration, improved signal-to-noise ratio (S/N) due 
to the increased XUV photon flux, and stronger streaking before the onset of the NIR field-induced ionization in attosecond streaking (2), or enhanced $S / N$ due to a reduced number of tunnelling steps in attosecond tunnelling spectroscopy (14).

Fig. 1 summarizes results of the modelling of the single-cycle interaction of ionizing NIR radiation with an ensemble of neon atoms (17). The left panels plot possible NIR electric waveforms, $E_{\mathrm{L}}(t)=E_{0} a_{\mathrm{L}}(t) e^{-i\left(\omega_{\mathrm{L}} l+\varphi\right)}+$ c.c., (c.c. stands for complex conjugate) derived from our streaking measurements (see Fig. 3) for different settings of the carrier-envelope (CE) phase, $\varphi$. Here, $E_{0}$ is the peak electric field strength, $a_{\mathrm{L}}(t)$ is the normalized complex amplitude envelope, and $\omega_{L}$ is the carrier frequency. The probability of ionization outside the central cycle is more than two orders of magnitude lower than that at the field maximum and hence negligible.

The spectra of XUV emissions originating from the individual recollisions (18) are predicted to differ by tens of electron volts in cut-off energy, and by up to orders of magnitude in intensity as a consequence of the single-cycle nature of the driving field. The strong variation of emission energies and intensities within a single wave cycle creates ideal conditions for isolated sub-100-as attosecond pulse generation. Indeed, filtering radiation with the bandpass depicted by the dashed-dotted line is predicted to isolate extreme ultraviolet radiation with more than $90 \%$ of its energy delivered in a single attosecond pulse for a range of CE phases as broad as $30^{\circ} \leq \varphi \leq 90^{\circ}$ (Fig. 1B). In contrast, with few-cycle-driven harmonic generation, resulting in isolated sub-fs pulses only over a relatively 
narrow range of the $C E$ phase near $\varphi \approx 0^{\circ}(3)$, single-cycle excitation appears to permit robust isolated attosecond pulses for a variety of driver waveforms, ranging from near-cosine to sine-shaped ones, owing to the order-of-magnitude variation of the ionization probability within a single wave cycle.

We used phase-controlled sub-1.5-cycle laser pulses carried at a wavelength of $\lambda_{\mathrm{L}}=c / \omega_{\mathrm{L}}=720 \mathrm{~nm}$ (19) to generate XUV harmonics in a neon gas jet up to photon energies of $\sim 110 \mathrm{eV}$ (fig. S1). The emerging XUV pulse following a spectral filtering through a bandpass (dash-dotted line in Fig. 1A) introduced by metal foils and a Mo/Si multilayer mirror (fig. S2) - subsequently propagates along with its NIR driver wave through a second jet of neon atoms, in which the XUV pulse ionizes the atoms in the presence of the NIR field. The freed electrons with initial momenta directed along the electric field vector of the linearly-polarized NIR field are collected and analyzed via time-of-flight (TOF) spectrometry (17).

The variation of the measured photoelectron spectra versus CE phase shows good agreement with the predictions of our simulations (Fig. 2A-B). Fig. $2 \mathrm{C}, \mathrm{D}, \mathrm{E}$ plot electron spectra corresponding to the CE phase settings selected in Fig. 1A. Apart from a downshift by the ionization potential of neon $(21.5 \mathrm{eV})$, they reveal close resemblance to the XUV spectra transmitted through the bandpass in Fig. $1 A(v),(v i),(i v)$, respectively. Panel $C$ depicts the broadest filtered spectrum produced by a single recollision (full-line spectrum in Fig. $1 A(v)$ ). Emission from the same recollision dominates also in the spectrum shown in Panel $E$, with this spectrum red-shifted and - upon transmission through the 
bandpass - correspondingly narrowed, as predicted in Fig. 1A(iv). The two humps of the spectrum plotted in Panel $D$ are indicative of contributions from two recollisions, in accordance with the "full-line" and "dotted-line" contributions to the emission spectrum in Fig. 1A(vi).

Before measuring the XUV pulse, we optimized the generation process by "fine-tuning" the NIR laser peak intensity in order to achieve the broadest possible XUV spectrum transmitted through the bandpass in the range of $\mathrm{CE}$ phase settings where the contrast is maximized $\left(50^{\circ}-80^{\circ}\right.$ according to Fig. 1B), to generate a clean single pulse with the shortest possible duration. For the temporal characterization of the generated XUV supercontinuum we shone the NIR field into the neon atoms ionized by the XUV pulse to implement the atomic transient recorder (ATR) technique introduced in $(3,4)$. The NIR field boosts or decreases the momenta of the photoelectrons, depending on their instant of release within the 2.4-fs period of the laser field, resulting in broadened and shifted (streaked) spectra of the electrons' final energy distribution. Fig. 3A plots a series of streaked spectra recorded versus delay between the XUV and NIR pulse, which we refer to as an ATR or streaking spectrogram. It is practically equivalent to the spectrogram obtained by frequency-resolved optical gating (FROG) (20), with the oscillating NIR field constituting an attosecond phase gate in the present case (21). As a consequence, a FROG retrieval algorithm (22) allows complete determination of both the (gated) XUV pulse and the (gating) NIR laser field (17). The reconstructed ATR spectrogram is plotted in Fig. 3B and reveals excellent agreement with the measured one. 
The retrieved temporal intensity profile and phase of the XUV pulse are shown in Fig. 3C. The pulse duration of $\tau_{\mathrm{x}}=80 \pm 5$ as is close to its transform limit of 75 as, with a small positive chirp of $\phi^{\prime \prime}=(1.5 \pm 0.2) \times 10^{3} a s^{2}$ being responsible for the deviation. As a further consistency check of the attosecond pulse retrieval, we compare the measured NIR-field-free electron spectrum (dashed line in Fig. 3D) with the electron spectrum calculated from the retrieved attosecond pulse (full line in Fig. 3D). Given that the pulse retrieval draws on streaked spectra that are strongly distorted with respect to the field-free one, the degree of agreement between the retrieved and directly measured spectrum provides yet another conclusive testimony of the reliability of the retrieved data.

The ATR retrieval algorithm indicates the presence of a satellite pulse accompanying the main attosecond pulse, containing $\sim 1 \%$ of the energy of the main pulse. This amount of satellite is is consistent with the depth of the experimentally observed modulation in the XUV spectrum (Fig. 3D). However, this result is inconsistent with our numerical modeling, which predicts a satellite energy content of some $6-7 \%$ for the optimum range of CE-phase settings (Fig. 1B). From analysis of the streaked spectra recorded at the maximum of the NIR electric field (fig. S3), where the momentum of the electrons released by the main attosecond pulse and its satellite is shifted in opposite directions, we infer a relative satellite energy of $\sim 8 \%$, in good agreement with the prediction of our modeling. As a consequence, the fringe visibility in the XUV spectrum is lower than implied by the relative amplitude of the satellite pulse. The discrepancy may originate from a temporal jitter between the main pulse and the satellite pulse, 
and/or from a different spatial amplitude distribution of the beams transporting the emission from the adjacent recollision events. The important lesson from these findings is that the fringe visibility in the XUV spectrum does not allow a reliable determination of the energy carried by the satellite(s) accompanying the main attosecond pulse.

The laser waveform evaluated from the ATR measurement is preserved between the location of the generation and measurement. In Fig. 4A we show the evaluated NIR waveform with electric field amplitude corresponding to a peak intensity of $I_{0} \sim(5.8 \pm 0.5) \times 10^{14} \mathrm{~W} / \mathrm{cm}^{2}$, as evaluated from the cut-off of our XUV spectra. The pulse duration (FWHM) of 3.3 fs is in good agreement with the results of previous interferometric autocorrelation measurements (19) and the evaluated CE phase of $\sim 50^{\circ}$ is consistent with the optimum contrast according to our modeling, see Fig. 1B.

Accurate knowledge of the attosecond XUV pulse parameters, the temporal evolution of the generating NIR field, and the emergence of the former from a single recollision permit one to perform precision tests of models of light-electron interactions underlying the ionization and subsequent attosecond pulse generation process. As an example, we have calculated the intrinsic spectral chirp, i.e. the variation of the group delay versus frequency, carried by the attosecond XUV pulse during its emergence from the chirp measured by the atomic transient recorder and the known dispersion of the components traversed by the pulse on its way from the source to the measurement (fig. S2). The result (dashed line in Fig. 4B) is compared with the intrinsic attosecond chirp (full line in 
Fig. 4B) calculated from a classical trajectory analysis $(23,24)$. There is a notable discrepancy at the high-energy components of the wavepacket, possibly due to quantum effects near cutoff. Nevertheless, the agreement with the attochirp resulting from short trajectories is stunning in the main part of the spectrum, where $S / N$ is excellent. This agreement indicates the powerfulness of semi-classical modeling of strong-field interactions $(25,26)$ and the negligible role of long trajectories in contributing to the XUV radiation in the far field (28).

In a similar way, the confinement of interaction between the ionizing field and the atom to a single wave cycle will permit accurate quantitative tests of theories of strong-field ionization. The sub-100 as XUV pulses emerging from the interaction with a flux greater than $10^{11}$ photons/s - along with their mono-cycle NIR driver wave - will push the resolution limit of attosecond spectroscopy to the atomic unit of time ( 24 as) and allow for the real-time observation of electron correlations, by means of streaking (6), tunneling (14), or absorption (27) spectroscopy. 


\section{References and Notes}

1. A. Baltuska et al., Nature 421,611 (2003).

2. R. Kienberger et al., Nature 427, 817 (2004).

3. E. Goulielmakis et al., Science 305, 1267 (2004).

4. M. F. Kling et al., Science 312,5771 (2006).

5. G. Sansone et al., Science 314,443 (2006).

6. A. Cavalieri et al., Nature 449, 1029 (2007).

7. E. Goulielmakis et al., Science 317, 769 (2007).

8. Resulting e.g. in shake-up, see: S. Svensson, B. Eriksson, N. Martensson, G. Wendin, U. J. Gelius, Electron Spectrosc. Rel. Phenom. 47, 327 (1988).

9. E.g. to ionizing radiation, see S. X. Hu, L. A. Collins, Phys. Rev. Lett. 96, 073004 (2006).

10. J. Breidbach, L. S. Cederbaum, Phys. Rev. Lett. 94, 033901 (2005).

11. A. I. Kuleff, J. Breidbach, L. S. Cederbaum, J. Chem. Phys. 123, 044111 (2005).

12. F. Remacle, R. D. Levine, PNAS 103, 6793 (2006).

13. G. Yudin, M. Y. Ivanov, Phys. Rev. A 64, 035401 (2001).

14. M. Uiberacker et al., Nature 446, 627 (2007).

15. T. Pfeifer et al., Phys. Rev. Lett. 97, 163901 (2006).

16. Y. Oishi et al., Opt. Expr. 14, 7230 (2006).

17. See supporting material on Science Online.

18. C. A. Haworth et al., Nature Phys. 3, 52 (2007).

19. A. L. Cavalieri et al., New. J. Phys. 9, 242 (2007).

20. R. Trebino, D. J. Kane, J. Opt. Soc. Am. A 10, 1101 (1993). 
21. Y. Mairesse, F. Quéré, Phys. Rev. A 71, 011401(R) (2005).

22. D. J. Kane, G. Rodriguez, A. J. Taylor, T. S. Clement, JOSA B 14, 935 (1997).

23. P. Salieres et al., Science 292, 902 (2001).

24. V. S. Yakovlev, A. Scrinzi, Phys. Rev. Lett. 91, 153901 (2003).

25. P. B. Corkum, Phys. Rev. Lett. 71, 1994 (1993).

26. K. J. Schafer, B. Yang, L. F. DiMauro, K. C. Kulander, Phys. Rev. Lett. 70, 1599 (1993).

27. Z.-H. Loh et al., Phys. Rev. Lett. 98, 143601 (2007).

28. R. López-Martens et al., Phys. Rev. Lett. 94, 033001 (2005).

29. K. Varjú et al., Laser Physics 15, 888 (2005).

30. Supported by the Max Planck Society and the DFG Cluster of Excellence: Munich Centre for Advanced Photonics (www.munich-photonics.de). E.G. acknowledges a Marie-Curie fellowship (MEIF-CT-2005-02440) and a MarieCurie Reintegration grant (MERG-CT-2007-208643). Andrew Aquila is supported by the NSF EUV ERC. 


\section{Figure Captions}

Fig. 1 Simulation of sub-fs XUV emission from neon atoms ionized by a linearlypolarized, sub-1.5-cycle, $720-\mathrm{nm}$ laser field. $E_{0}$ and $a_{\mathrm{L}}(t)$ are inferred from best agreement between the modelled (17) and measured (Fig. 2) spectra and the streaking spectrogram (Fig. 3), respectively. The laser field liberates electrons near its most intense wave crests. (A) The classical trajectories of maximum return energy (left panels) and the spectra of emerging XUV emission (right panels) are shown for waveforms consistent with $a_{\mathrm{L}}(t)$ inferred from Fig. 3 (correspondence established by colors and line style). The numbers in panels (i)-(iii) quantify, in units of $10^{-4}$, the ionization probability and hence the squared modulus of the amplitude of the electron wavepackets launched. This amplitude substantially dictates the intensity of XUV emission upon recollision, contrast ionization probability in panels (i)-(iii) with corresponding emission intensities in panels (iv)-(vi). The "dotted-line" emission is not visible in panel $(v)$ because of the lower ionization probability (by two orders of magnitude) with respect to that resulting in the "full-line" emission, see panel (ii). Dashed-dotted line: bandpass used in our experiments (fig. S2). $\varphi=70^{\circ}$ and $\varphi=135^{\circ}$ yield highest contrast, see (B), and highest XUV cutoff energy, respectively. (B) Contrast, defined as the ratio of the energy of the main attosecond XUV pulse to the overall XUV emission energy transmitted through the bandpass (dash-dotted line), versus CE-phase.

Fig. 2 Control of bandpass-filtered XUV emission with the waveform of mono-cycle light. Measured $(A)$ and simulated (B) (17) photoelectron spectra versus CE phase, with the 
delay increased in steps of $\sim 11$ degrees $(\pi / 16)$. (C), (D), (E) Spectra measured at the CE phase setting closest to the values selected in Fig. $1 \mathrm{~A}$. The zero of the CE phase scale in (A) was set to yield the best agreement with the modeled spectra in (B).

Fig. 3 Sub-100 as XUV pulse retrieval. (A) Measured ATR spectrogram compiled from 126 energy spectra of photoelectrons launched by an XUV pulse with a bandwidth of $\sim 28 \mathrm{eV}(\mathrm{FWHM})$ and recorded at delay settings increased in steps of 80 attoseconds. Here, a positive delay corresponds to the XUV pulse arriving before the NIR pulse. The high flux of the XUV source allows this spectrogram to be recorded within some 30 minutes. (B) ATR spectrogram reconstructed after some $10^{3}$ iterations of the FROG algorithm (17). (C) Retrieved temporal intensity profile and spectral phase of the XUV pulse. The intrinsic chirp of the XUV emission (see Fig. 4B) is almost fully compensated by a 300-nm-thick $\mathrm{Zr}$ foil introduced into the XUV beam between the attosecond source and the ATR measurement. (D) XUV spectra evaluated from the measurement of the XUV-generated photoelectron spectrum in the absence of the NIR streaking field (dashed line) and from the ATR retrieval (full line). Dotted line, retrieved spectral phase.

Fig. 4 (A) Retrieved electric field of the NIR laser pulse used for generating and measuring the attosecond XUV pulse shown in Fig. 3. The duration of the pulse (full width at half maximum of the cycle-averaged intensity profile) is $\tau_{\mathrm{L}}=3.3 f \mathrm{~s}$, the CE phase is evaluated as $\varphi \sim 50^{\circ}$. The classical electron trajectories responsible for the emission of the filtered attosecond pulse are calculated by using the plotted electric field and shown in the same panel. The color coding indicates the final return energy of the electrons. (B) Energy of the recolliding electron plus ionization potential (= emitted XUV photon energy) versus recollision time evaluated from the classical trajectory analysis 
(full green line), emitted photon energy versus emission time (dashed purple line) inferred from the chirp of the measured attosecond pulse and the dispersion of the metal filter which the attosecond pulse passes through before the measurement. The basic idea for the graphical representation is borrowed from (29).

Figures 

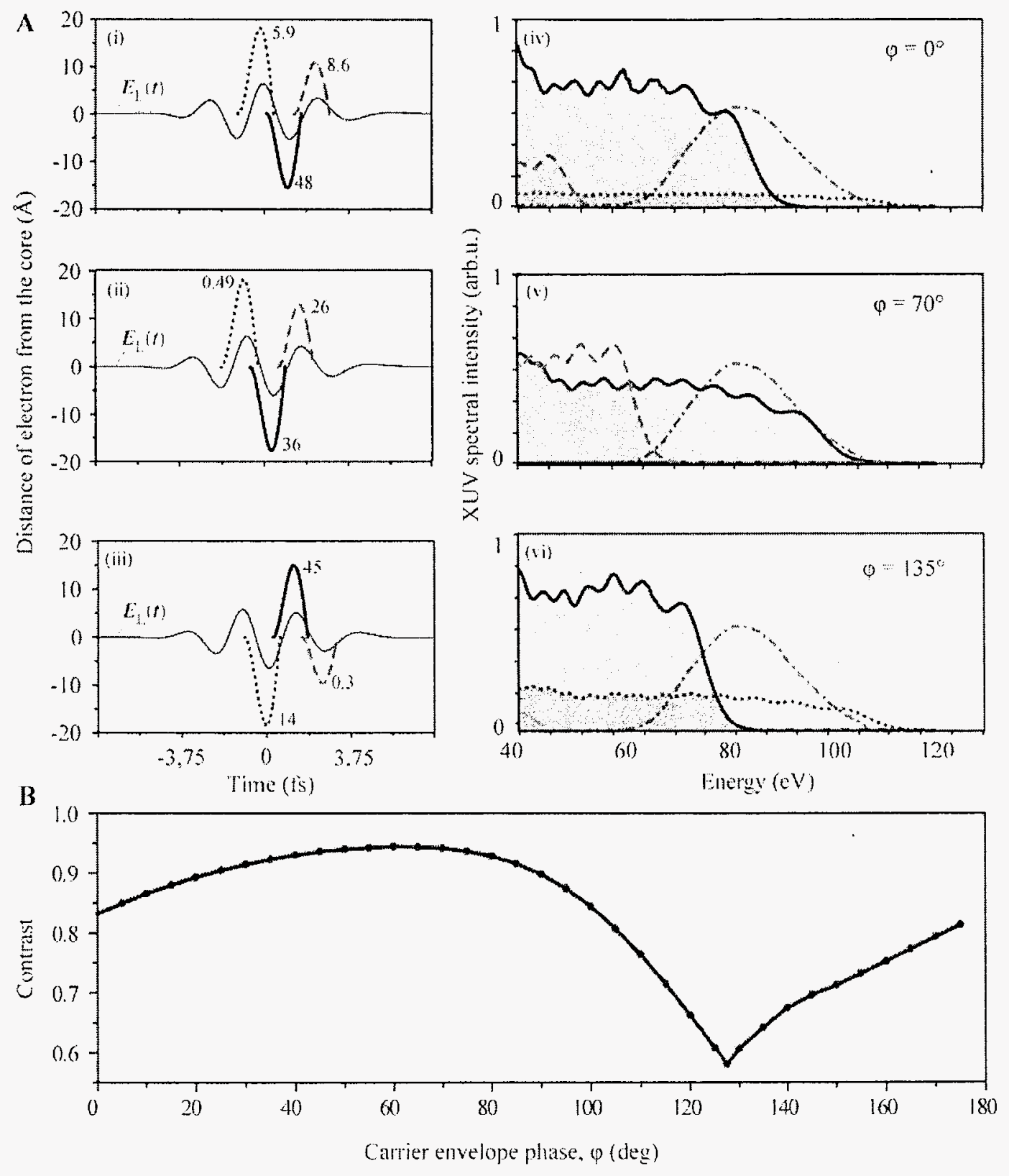

Figure 1 

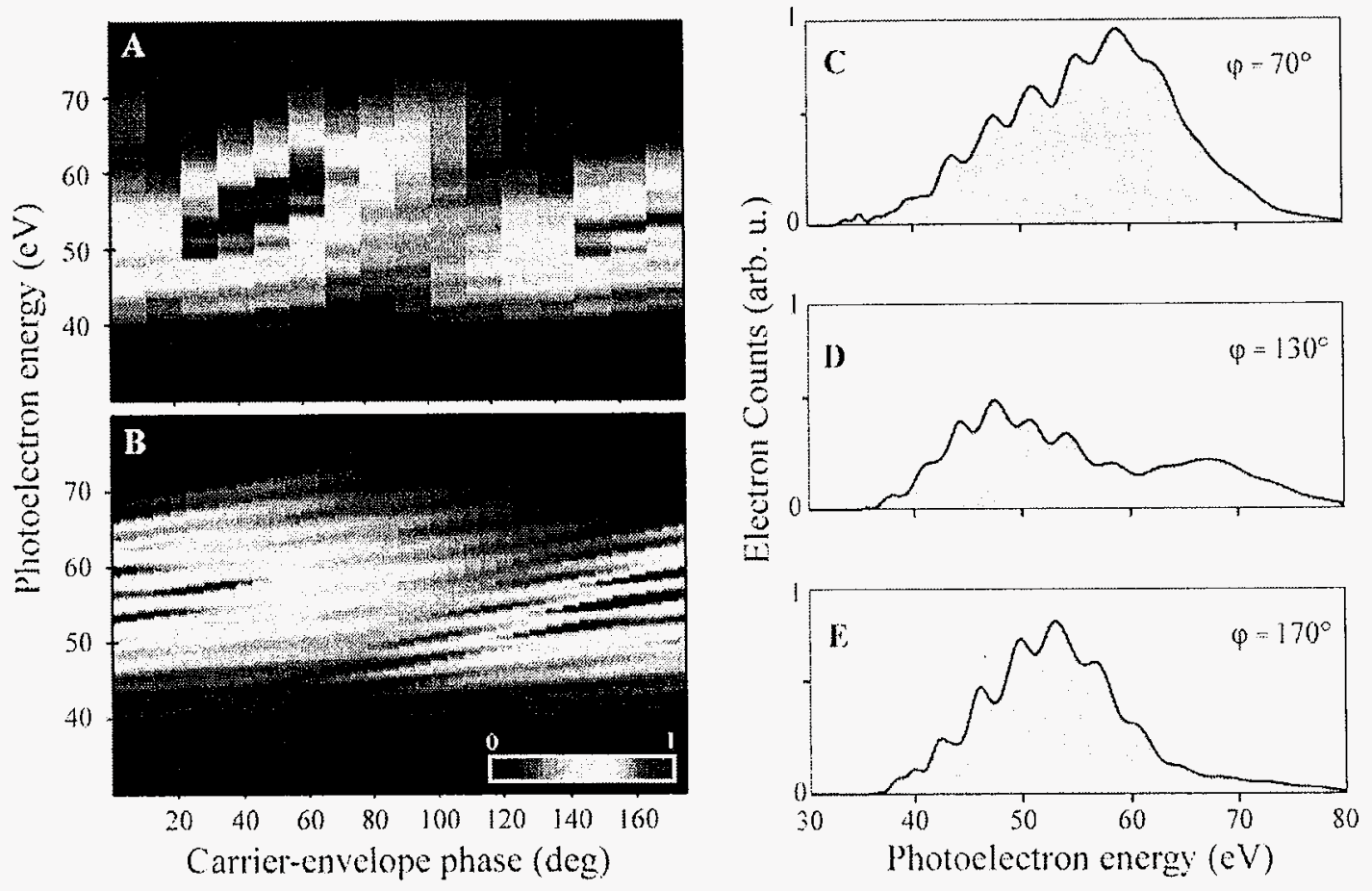

Figure 2 

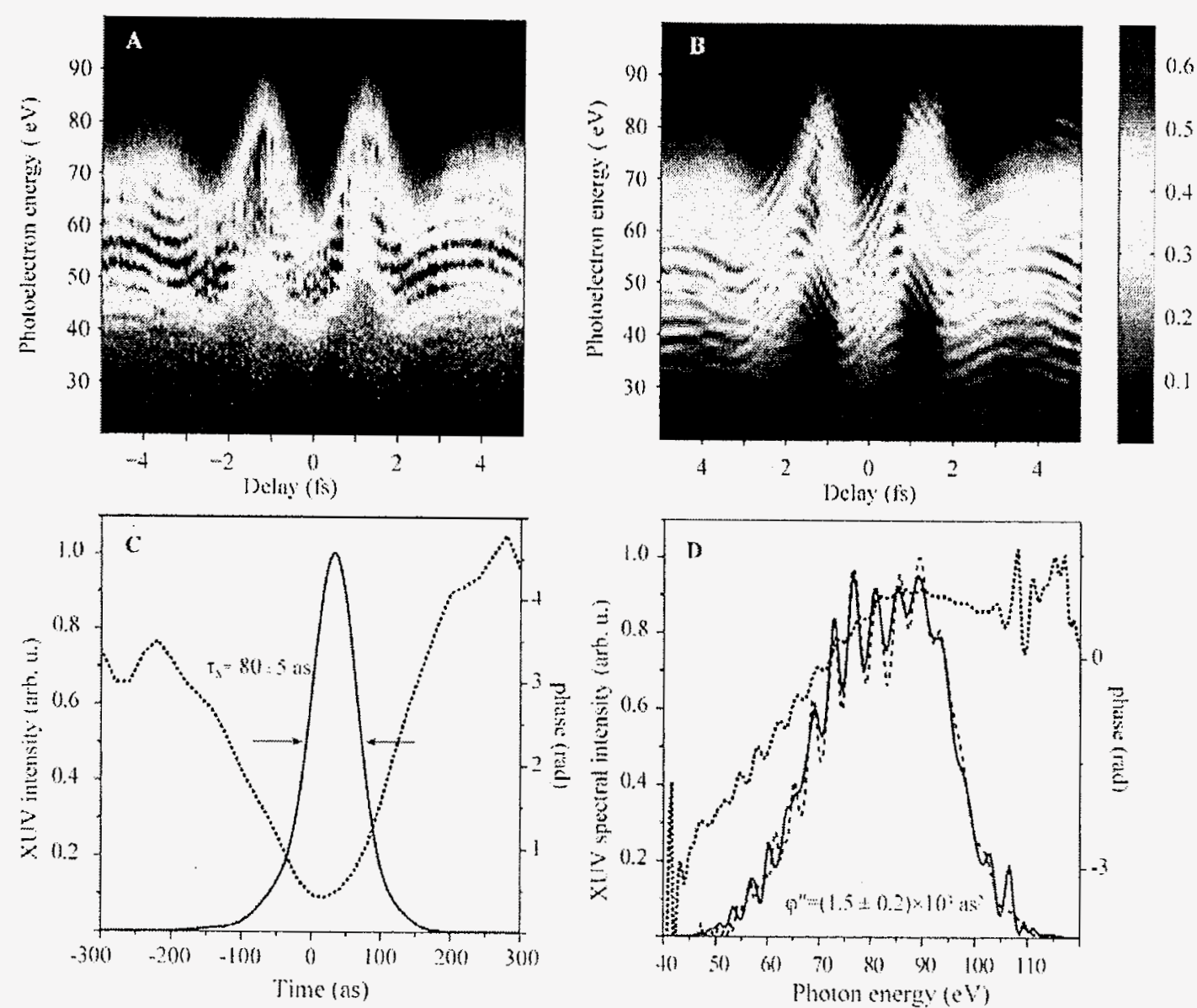

Figure 3 


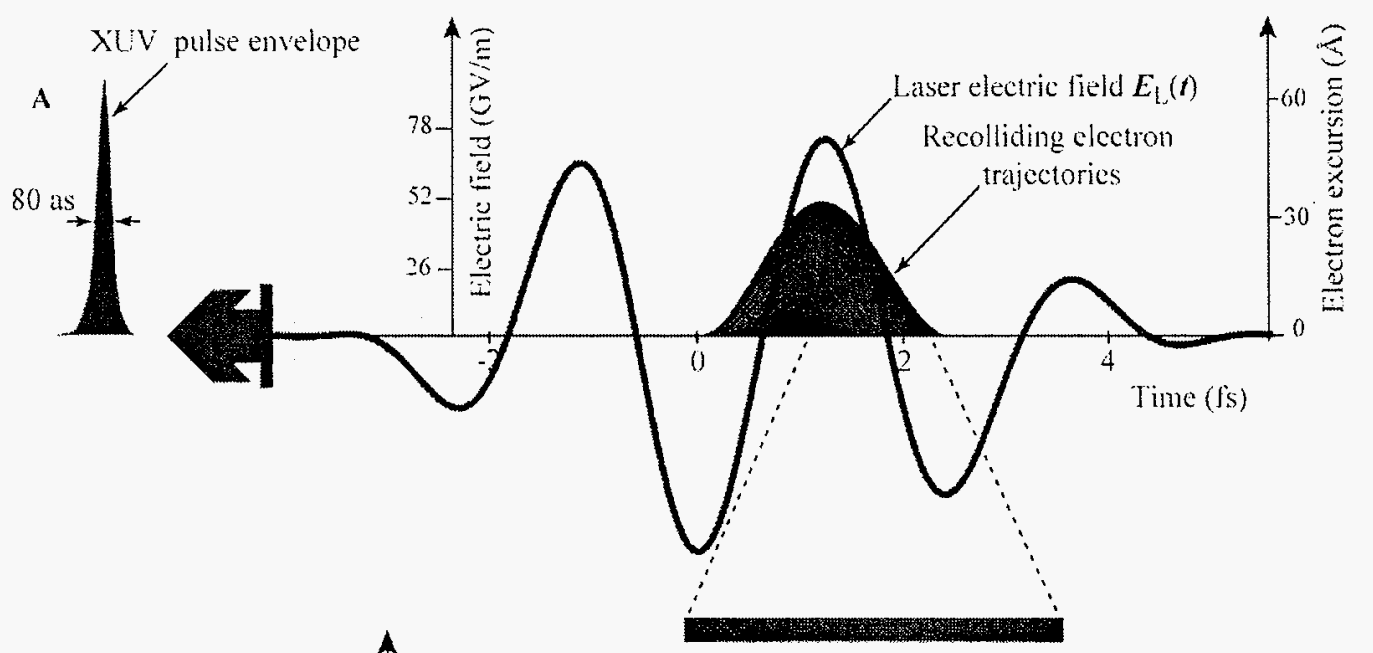

B

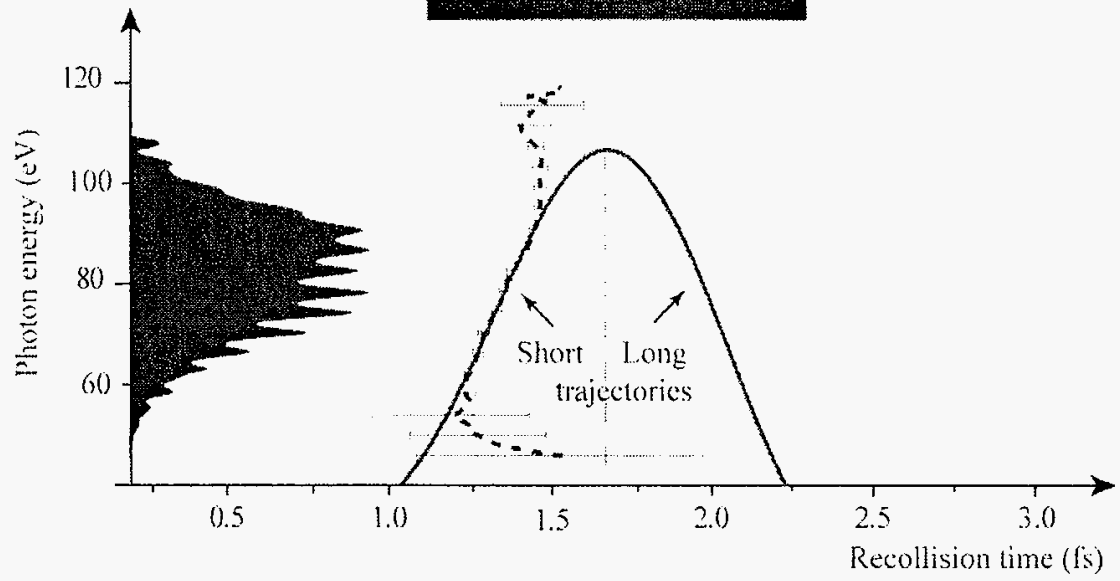

Figure 4 
